Original Article

\title{
Comparisons of changes in the two-point discrimination test following muscle fatigue in healthy adults
}

\author{
Jintae Han, PT, $\mathrm{PhD}^{1)}$, Soojin Park, PT, PhD ${ }^{1)^{*}}$, Seonghyun Jung ${ }^{1)}$, Yeounsung Choi ${ }^{1)}$, \\ Hyunjoo Song ${ }^{1)}$ \\ 1) Department of Physical Therapy, College of Science, Kyungsung University: 309 Suyeong-ro, \\ Nam-gu, Busan 608-736, Republic of Korea
}

\begin{abstract}
Purpose] This study aimed to examine the effects of muscle fatigue on skin sensation by looking at changes in two-point discrimination (2PD) after inducing muscle fatigue. [Subjects] Thirty-four young and healthy adults with no pathological symptoms (17 males; 17 females) participated in this study. [Methods] Continuous isometric contraction was applied to the upper and lower extremities with an intensity of $50 \%$ of the maximal muscle strength to trigger muscle fatigue, and then the $2 \mathrm{PD}$ test was conducted on the $3 \mathrm{~cm}$ medial area of the elbow and $5 \mathrm{~cm}$ area of the knee bone. [Results] After muscle fatigue was induced, the 2PD distance significantly increased in the upper and lower extremities of both males and females. Before triggering muscle fatigue, the 2PD distance was longer in males than females, but after causing muscle fatigue, there was no difference between males and females. The increase in 2PD distance after the experiment was more significant in females than males. [Conclusion] Muscle fatigue has an effect of reducing the skin's sensory faculty by increasing the 2PD distance in the skin. There is a difference in the response of the skin according to body area and gender; therefore, caution is needed to avoid triggering fatigue during exercise.

Key words: Muscle fatigue, Skin sensation, Two-point discrimination
\end{abstract}

(This article was submitted Jul. 22, 2014, and was accepted Aug. 24, 2014)

\section{INTRODUCTION}

Sensory information about human movements is obtained by comprehensively processing proprioceptive sensations provided by cutaneous afferent sensory organs as well as articular mechanoreceptors and muscle receptors ${ }^{1)}$. Humans maintain an appropriate posture and perform precise motions based on information sent by proprioceptive sensory organs. Among the different senses, the tactile sense provides sensory information that flows from the external environment's stimulating sensory organs within the skin. It works as a protection mechanism against harmful stimuli, enables communications between the external environment and the human body, and plays an important role in postural control and maintenance during human movement and work ${ }^{2}$.

However, the sensations are influenced by age ${ }^{3)}$, weight ${ }^{4)}$, skin temperature ${ }^{5)}$, disease ${ }^{6)}$, muscle fatigue ${ }^{7)}$, and gender $^{8)}$. Among the factors that affect sensation, muscle fatigue that occurs due to muscle contraction activity lowers position sense ability, possibly triggering problems with motor and

*Corresponding author. Soojin Park (E-mail: rememversj@ hanmail.net)

C2015 The Society of Physical Therapy Science. Published by IPEC Inc. This is an open-access article distributed under the terms of the Creative Commons Attribution Non-Commercial No Derivatives (by-ncnd) License $<$ http://creativecommons.org/licenses/by-nc-nd/3.0/> postural control capabilities ${ }^{9)}$. It is known that during isometric exercise, the intramuscular temperature goes up; as a result, afferent sensory fibers of the muscle spindles and the Golgi tendon organs are activated, and activity of the secondary sensory ending is reduced or stopped, leading to errors in proprioceptive sensation and as a result decreasing tactile sense sensitivity in the skin ${ }^{10)}$.

Many studies have evaluated changes in proprioceptive senses using a joint repositioning ability test ${ }^{1,11)}$ and a force reproduction test ${ }^{10)}$ of certain muscles after triggering muscle fatigue through muscle contraction exercises. These studies have limitations in that they focused on changes in sensory receptor and motor neuron activation, and such tests are difficult to conduct. On the other hand, among the different methods used to evaluate proprioceptive sensation, two-point discrimination (2PD) is a technique that measures tactile spatial acuity ${ }^{12)}$. It is not difficult to take a precise and reliable measurement with this method; therefore, it is widely used to assess proprioceptive sensation ${ }^{8)}$. As a kind of cutaneous sensory assessment technique, 2PD is an essential evaluation method to test the sensory ability of patients with peripheral afferent nerve injury ${ }^{13)}$. Many clinicians use it in order to judge sensory perception ability and changes by measuring intracutaneous sensory innervation density and functional tactile sensitivity of the distal segments ${ }^{14}$ ).

Currently, there are not many studies that have examined the effects of muscle fatigue on cutaneous tactile sense. Accordingly, the aim of this study was to examine the effects 
of muscle fatigue on skin sensation by looking at changes in 2PD after triggering muscle fatigue using isometric exercises of the upper and lower extremities.

\section{SUBJECTS AND METHODS}

Thirty-four young and healthy adults (17 males; 17 females) participated in this study. They had no musculoskeletal system disease, skin disease, scars, burns, or dermal hypersensitivity. All subjects read and signed consent forms in accordance with the ethical standards of the Declaration of Helsinki. In order to cause muscle fatigue of the upper and lower extremities, an mtd feedback ball system (MTD Systems LLC, Neunburg v. Wald, Germany) was used to measure maximal muscle strength of the upper and lower limbs. The mtd feedback ball system is a motoric rehabilitation tool, and it offers the ability to perform measurement and training of various individual functions up to complex ADL functions in physical therapy. Then the subjects were instructed to maintain $50 \%$ of maximal muscle strength. The point at which they failed to maintain $50 \%$ of maximal muscle strength was designated as the point when muscle fatigue was triggered. A $2 \mathrm{PD}$ test was carried out using an aesthesiometer (Instrument Company, Lafayette, IN, USA), a tool for a 2PD test, right after muscle fatigue was induced. The test was conducted while the subjects closed their eyes. The shortest for which two points could be felt was considered the measured value for the 2PD. When the biceps muscle of the upper extremities was tested, the location was the $3 \mathrm{~cm}$ medial area of the elbow. The rectus femoris muscle of the lower extremities was also tested, and a $5 \mathrm{~cm}$ area of the knee bone was measured. Measurements were taken three times for each test, and average values were used for analysis.

A Wilcoxon test was used to examine 2PD differences between before and after fatigue, and a Mann-Whitney test was performed to compare differences in 2DP between the two groups before and after fatigue. An alpha level of 0.05 was used to test for significance. All data were analyzed using the SPSS 21.0 statistical software.

\section{RESULTS}

The 2PD perception distance significantly increased in both males and females after including muscle fatigue $(p<0.05)$ (Tables 1 and 2$)$, which means the sensitivity of skin sensation decreased after induction of muscle fatigue. The 2PD perception distance of male subjects was significantly longer than that of female subjects $(p<0.05)$. After muscle fatigue was triggered, the $2 \mathrm{PD}$ perception distance of the male subjects was longer than that of their female counterparts, but there was no significant difference between them ( $>>0.05$ ) (Table 1). The 2PD perception distance of the upper and lower extremities significantly increased after muscle fatigue was induced $(\mathrm{p}<0.05)$ (Table 2); however, the 2PD perception distance of the lower extremities was significantly longer than that of the upper extremities before and after induction of muscle fatigue $(\mathrm{p}<0.05)$ (Table 2$)$. The 2PD perception distance increased more significantly in females than males after muscle fatigue was triggered
Table 1. Comparison of distance of $2 \mathrm{PD}$ between males and females before and after fatigue (mean \pm SD)

units: $\mathrm{mm}$

\begin{tabular}{llcr}
\hline Variable & Time & Upper limb & Lower limb \\
\hline \multirow{2}{*}{ Distance of 2PD } & Before & $42.8 \pm 11.1$ & $31.2 \pm 11.1^{\mathrm{a}}$ \\
& After & $56.29 \pm 12.3^{\mathrm{b}}$ & $50.24 \pm 13.8^{\mathrm{b}}$ \\
\hline
\end{tabular}

${ }^{a}$ Significant difference between males and females $(p<0.05)$

${ }^{\mathrm{b}}$ Significant difference between before and after fatigue $(\mathrm{p}<0.05)$

2PD: two-point discrimination

Table 2. Comparisons of distance of 2PD between the upper and lower limbs before and after fatigue $(\operatorname{mean} \pm \mathrm{SD})$

units: $\mathrm{mm}$

\begin{tabular}{cllc}
\hline Variable & Time & Upper limb & Lower limb \\
\hline \multirow{2}{*}{ Distance of 2PD } & Before & $29.9 \pm 9.0$ & $44.1 \pm 11.4^{\mathrm{a}}$ \\
& After & $45.3 \pm 10.5^{\mathrm{b}}$ & $61.2 \pm 10.7^{\mathrm{ab}}$ \\
\hline
\end{tabular}

a Significant difference between the upper limb and lower limbs $(\mathrm{p}<0.05)$. ${ }^{\mathrm{b}}$ Significant difference between before and after fatigue $(\mathrm{p}<0.05)$

2PD: two-point discrimination

Table 3. Comparisons of the changes of $2 \mathrm{PD}$ between groups following fatigue (mean $\pm \mathrm{SD}$ )

units: $\mathrm{mm}$

\begin{tabular}{lcc}
\hline Variable & \multicolumn{2}{c}{ Sex or Limb } \\
\hline & Male & Female \\
Changes of 2PD & $13.5 \pm 3.5$ & $19.0 \pm 7.0^{*}$ \\
& Upper limb & Lower limb \\
& $15.4 \pm 4.7$ & $17.2 \pm 7.3$ \\
\hline
\end{tabular}

*Significant difference between groups $(\mathrm{p}<0.05)$

2PD: two-point discrimination

$(\mathrm{p}<0.05)$. The changes in the 2PD perception distance of the lower extremities were greater than those of the upper extremities, but they did not significantly differ $(p>0.05)$ (Table 3). This shows that sensitivity of females to skin sensations decreases more than that of males after the induction of muscle fatigue.

\section{DISCUSSION}

Cutaneous sensory assessment is a general neurologic examination that evaluates the function of peripheral sensory nerves and appraises temperature, pressure, light touch, and vibration. In particular, light tough stimulates two points so as not to trigger discomfort on the skin, and the sensory ability of the skin is evaluated for light touch using the 2PD test, which estimates the ability to discriminate the distance between two points ${ }^{13)}$

In the present study, how muscle fatigue influences upper and lower extremity skin sensation of males and females was examined using 2PD. Using healthy adults in their 20s as the subjects, this study applied continuous isometric contraction and triggered muscle fatigue, and it then compared the 
result of 2PD tests conducted before and after muscle fatigue induction. The results showed that both male and female subjects saw the 2PD perception distance of their upper and lower limbs significantly increase after muscle fatigue was triggered.

The reason why muscle fatigue reduces the sensitivity of 2PD on the skin is because the signal transmission speed goes down in the muscle spindle (the intramuscular motor system), the Golgi tendon organ, and the intracutaneous somatosensory system. As a result, the time taken for sensory information to be input into the central nervous system and trigger a response is delayed ${ }^{1)}$, and changes in information input from such sensory organs give rise to errors in the information processing procedures in the central nervous system $^{15}$. Such changes affect proprioceptive sensation perception, triggering disability in position sense perception in the skin, influencing postural control ${ }^{15)}$, and possibly triggering problems with skin protection mechanisms ${ }^{13)}$.

Shields et al. ${ }^{1)}$ reported that muscle fatigue in the ankle dorsiflexor decreased the repositioning ability of the ankle, and Lee and $\mathrm{Ha}^{10)}$ noted that muscle fatigue resulting from isometric contraction considerably reduced the force reproduction ability of the elbow flexors compared with the ability before muscle fatigue induction. They observed that these phenomena were due to proprioceptive hypoesthesia caused by muscle fatigue adversely affecting the motor control ability of the joints.

Nolan ${ }^{13)}$ performed the 2PD test on the upper limbs of healthy males and females in their 20 s and compared the results. The forearm was more sensitive than the upper arm, and the 2PD values of the males were lower than those of the females. The palmar surface of the hand showed considerably higher sensitivity than other areas of the upper limb. In a study by Lee ${ }^{8)}, 2 \mathrm{PD}$ sensitivity varied among different body parts; $2 \mathrm{PD}$ was short in the shoulder bone, the thigh, the lower arm, the top of the foot, and the top of the hand. In the present study, sensitivity also differed according to different body areas; $2 \mathrm{PD}$ was relatively greater in the lower limb than in the upper limb both prior to and after muscle fatigue induction. This is because the upper limbs are areas that perform more precise movements than the lower limbs; therefore, they have a higher sensory organ density than the lower limbs. In this regard, Nolan ${ }^{13)}$ noted that differences in sensitivity according to different body parts were due to individual differences rather than embryological differences or the spinal cord nerve sensitivity of certain areas. The reason for individual differences has not yet been elucidated.

The present study also identified changes in 2PD according to gender. Before muscle fatigue was triggered, the $2 \mathrm{PD}$ distance of the males was significantly longer than that of the females, but after muscle fatigue was induced, there was no difference between them. When the changes after muscle fatigue induction were compared between the two genders, the changes of the female subjects were relatively larger than those of the male subjects, and sensitivity to fatigue was greater in females than males. Nolan ${ }^{13)}$ compared 2PD between males and females and found that the medial surface of the forearm of males was statistically significantly more sensitive than that of females, but there was no difference between them in other areas. According to $\mathrm{Lee}^{8)}$, the 2PD ability of females was better that that of males in the shoulder bone, the top of the hand, and the top of the foot; there was no significant difference between them in the lower arm and thigh.

Muscles have different nerve signal transmission speeds and varying action potentials in motor units due to muscle fiber type. The rate of type 1 muscle fibers is higher in females than males, and the rate of type 2 muscle fibers is higher in males than females. The cross-sectional areas of fibers are larger in males than females, action potential intensity tends to be lower in females than males, torque induction ability is better in males than females, and resistance to muscle contraction fatigue is stronger in females than males ${ }^{16)}$.

In the present and previous studies, 2PD sensitivity differed according to gender. Overall, this is due to anatomophysiological differences in male and female muscles, higher skin sensitivity in females than males, and environmental changes affecting females more than males.

In general, a warming-up exercise ${ }^{17}$ ) and a moderate muscular exercise ${ }^{18)}$ are known to improve proprioceptive sensation. As shown in previous studies, however, exercise that causes fatigue in the muscles lowers proprioceptive sensory function, negatively affecting physical control. The decrease in 2PD capabilities in the present study led to lower pressure sense perception ability ${ }^{8)}$, which may trigger problems with object perception ability using tactile senses as well as postural control ability due to problems with weight perception by the lower extremities ${ }^{19}$ ). Therefore, when exercise is prescribed for patients whose proprioceptive sensation has been lowered, an exercise regimen that does not trigger fatigue should be applied in consideration of differences in sensory perception ability according to age, gender, and body part. In addition, additional research to discover anatomo-physiological causes of cutaneous sensibility differences according to gender is considered necessary.

\section{ACKNOWLEDGEMENT}

This research was supported by a Kyungsung University Research Grant in 2015.

\section{REFERENCES}

1) Shields RK, Madhavan S, Cole K: Sustained muscle activity minimally influences dynamic position sense of the ankle. J Orthop Sports Phys Ther, 2005, 35: 443-451. [Medline] [CrossRef]

2) Jones LA, Sarter NB: Tactile displays: guidance for their design and application. Hum Factors, 2008, 50: 90-111. [Medline] [CrossRef]

3) Ribeiro F, Oliveira J: Effect of physical exercise and age on knee joint position sense. Arch Gerontol Geriatr, 2010, 51: 64-67. [Medline] [CrossRef]

4) Paschalis V, Nikolaidis MG, Theodorou AA, et al.: The effects of eccentric exercise on muscle function and proprioception of individuals being overweight and underweight. J Strength Cond Res, 2013, 27: 2542-2551. [Medline] [CrossRef]

5) Ribeiro F, Moreira S, Neto J, et al.: Is the deleterious effect of cryotherapy on proprioception mitigated by exercise? Int J Sports Med, 2013, 34: 444448. [Medline]

6) Piriyaprasarth P, Morris ME, Delany C, et al.: Trials needed to assess knee proprioception following stroke. Physiother Res Int, 2009, 14: 6-16. [Medline] [CrossRef]

7) Riemann BL, Lephart SM: The sensorimotor system, part II: The role of proprioception in motor control and functional joint stability. J Athl Train, 2002, 37: 80-84. [Medline]

8) Lee JW: Correlation between static two-point discrimination and passive 
sponge softness discrimination in health adult. J Neurocog Rehabil, 2009, 1: $1-10$.

9) Choi BO, Kim MW, Hong HS: The effect of lower extremity muscula fatigue on proprioceptive position sense of the knee joint. J Kor Soc Sports Phys Ther, 2009, 5: 19-25.

10) Lee WH, Ha SM: Influence of muscle fatigue on the sensing of force reproduction in elbow flexors. Phys Ther Kor, 2007, 14: 16-22.

11) Kwon OS, Lee SW, Seo DK, et al.: The effect of exercise-induced fatigue on knee joint position sense in the young, elderly adults and stroke patients. J Korean Soc Phys Med, 2013, 8: 619-625. [CrossRef]

12) Tong J, Mao O, Goldreich D: Two-point orientation discrimination versus the traditional two-point test for tactile spatial acuity assessment. Fron Hum Neurosci, 2013, 7: 579. [Medline] [CrossRef]

13) Nolan MF: Two-point discrimination assessment in the upper limb in young adult men and women. Phys Ther, 1982, 62: 965-969. [Medline]

14) Franco PG, Bohrer RC, Rodacki AL: Intra-observer reproducibility of the feet soles two-point discrimination test in asymptomatic elderly and young individuals. Rev Bras Fisioter, 2012, 16: 523-527. [Medline] [CrossRef]

15) Gandevia SC: Spinal and supraspinal factors in human muscle fatigue. Physiol Rev, 2001, 81: 1725-1789. [Medline]

16) von Tscharner V, Goepfert B: Gender dependent EMGs of runners resolved by time/frequency and principal pattern analysis. J Electromyogr Kinesiol, 2003, 13: 253-272. [Medline] [CrossRef]

17) Lim KI, Hwnagbo G, Nam HC, et al.: Comparison of the effects on dynamic balance ability of warming uo in water versus on the ground. J Phys Ther Sci, 2014, 26: 575-578. [Medline] [CrossRef]

18) Kim CS, Gong WT, Kim SG: The effect of lower extremity muscle strengthening exercise and treadmill walking exercise on the gait and balance of stroke patients. J Phys Ther Sci, 2011, 23: 405-408. [CrossRef]

19) Song BK: The effect of tactile stimulation on two point discrimination, hand function, and ADL in impaired characteristics of stroke patient. J Korean Soc Phys Med, 2012, 7: 481-491. [CrossRef] 